\title{
A POLYMER CONDENSER MICROPHONE ON SILICON WITH ON-CHIP
} CMOS AMPLIFIER

\author{
Michael Pedersen, Wouter Olthuis \& Piet Bergveld \\ MESA Research Institute, University of Twente \\ P.O. Box 217, NL-7500 AE Enschede, the Netherlands \\ Phone: +31 53 4892722, Fax: +31534892287 \\ e-mail: M.Pedersen@eltn.utwente.nl
}

\begin{abstract}
SUMMARY
In this paper the development of a capacitive microphone with integrated preamplifier is described. The condenser microphone is made by micromachining of polyimide on silicon, and is compatible with CMOS technology. Therefore, the structure can be realised by post processing on substrates containing integrated circuits, independently of the IC process. Microphones with a required $\mathrm{DC}$ bias voltage of $4 \mathrm{~V}$ have been realised on a CMOS substrate containing PMOS buffer preamplifiers. From the measurements on these structures, it is illustrated how an immediate improvement of $4.8 \mathrm{~dB}$ of the microphone sensitivity and noise level can be obtained by using the integrated preamplifier. The measured sensitivity of the integrated condenser microphone was $2.5 \mathrm{mV} / \mathrm{Pa}$ and the equivalent noise level (ENL) was $29.5 \mathrm{~dB}(\mathrm{~A}) \mathrm{SPL}$.
\end{abstract}

\section{INTRODUCTION}

The evolution of a new generation of small-size highperformance microphones based on silicon micromachining technology has until now been focused on the need to monolithically integrate the sensor structure with an electronic circuit to maximise the performance. Since most sensor fabrication processes are incompatible with IC processes, new developments have been required to attain a reliable, noninterfering and simple sensor process, which can be performed after completion of the integrated circuit. For this purpose, the sensor structure shown in figure 1 has been developed [1]. As opposed to more conventional silicon sensors, this sensor is a plastic (polyimide) structure made directly on the substrate by micromachining. The advantage of this structure is the lowtemperature $\left(<300{ }^{\circ} \mathrm{C}\right)$ fabrication process, which can be performed on substrates containing integrated circuits without inflicting any damage.

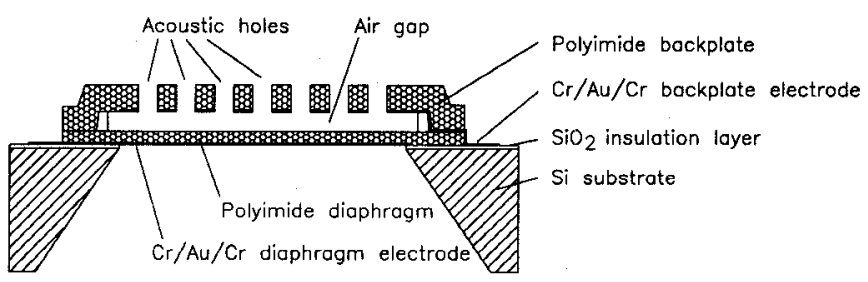

Figure 1: An IC-compatible polyimide condenser microphone.

\section{FABRICATION TECHNOLOGY}

The fabrication process on substrates with circuits made with a twin-well CMOS process is shown in figure 2. Firstly, the $\mathrm{Cr} / \mathrm{Au} / \mathrm{Cr}$ diaphragm electrode is deposited and patterned using lift-off with photoresist. This ensures that the metal never is in direct physical contact with the surface of the integrated circuit.
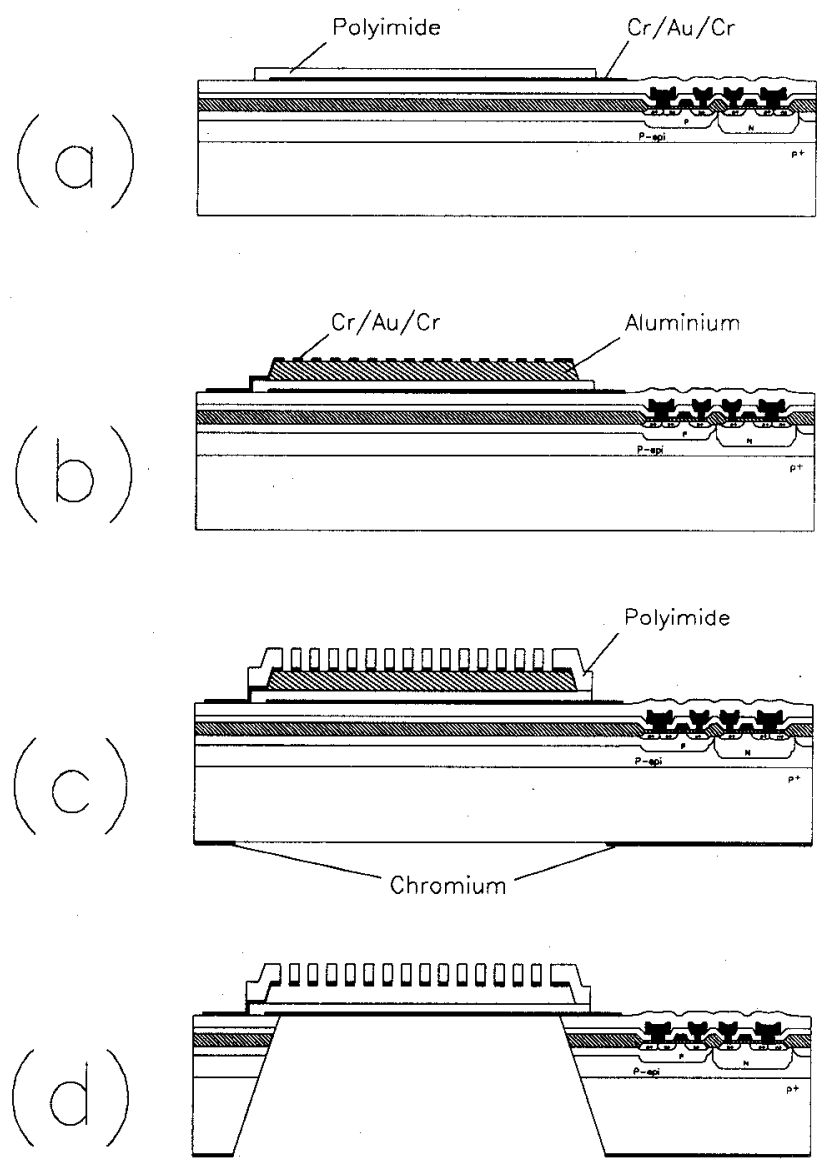

Figure 2: Fabrication process of the integrated polyimide condenser microphone.

Thereafter, the first polyimide layer is spun onto the substrate, patterned and cured at $300{ }^{\circ} \mathrm{C}$, yielding the structure in figure 2(a). The polyimide (HTR3 from OCG Microelectronics) is photosensitive and in liquid form, and may therefore be processed as conventional photoresist. Subsequently, the $\mathrm{Al}$ 
sacrificial layer is deposited and patterned, during which the integrated circuit is protected with photoresist, and a second $\mathrm{Cr} / \mathrm{Au} / \mathrm{Cr}$ electrode is deposited and patterned (figure 2(b)). After applying and curing the second polyimide layer, a $\mathrm{Cr}$ etch mask is deposited and patterned on the backside of the substrate (figure 2(c)). The sacrificial Al layer is then etched to realise the air gap, and finally the substrate is etched from the backside using reactive ion etching, in which the $\mathrm{Cr} / \mathrm{Au} / \mathrm{Cr}$ diaphragm electrode also serves as en etch-stop (figure 2(d)). The realised microphones have a diaphragm size and thickness of $2 \mathrm{~mm}$ and $1 \mu \mathrm{m}$, an air gap of $2 \mu \mathrm{m}$, and a backplate thickness of $17 \mu \mathrm{m}$.

\section{MEASUREMENTS AND RESULTS}

Microphones have been integrated with a CMOS preamplifier, the gain and bandwidth of which was measured to be $-0.75 \mathrm{~dB}$ and $180 \mathrm{kHz}$. In figure 3 , a photograph of a substrate containing integrated condenser microphones and preamplifiers is shown. The microphone has been characterised in an acoustical set-up, in which the frequency response may be measured and compared to that of a reference microphone [2]. The frequency response of the condenser microphone was measured both with the integrated preamplifier $(\mathbf{A})$ and with an external preamplifier in the measurement setup $(\mathbf{B})$. From the results shown in figure 4 , it can be seen that the measured sensitivity of the microphone increases by $4.8 \mathrm{~dB}$ if the integrated amplifier is used, which is due to the reduced parasitic loading of the microphone. The A-weighted noise at output of the preamplifier was measured in both situations to be $1.5 \mu \mathrm{V}$ for a bandwidth of $200 \mathrm{kHz}$. Therefore, the $4.8 \mathrm{~dB}$ increase of the output signal with the integrated amplifier gives a direct improvement of the ENL by $4.8 \mathrm{~dB}$. The ENL was calculated to be $34.3 \mathrm{~dB}(\mathrm{~A})$ SPL with the external amplifier and $29.5 \mathrm{~dB}(\mathrm{~A})$ SPL with the integrated amplifier, which is more than sufficient to detect normal human speech (50-70 dB SPL).

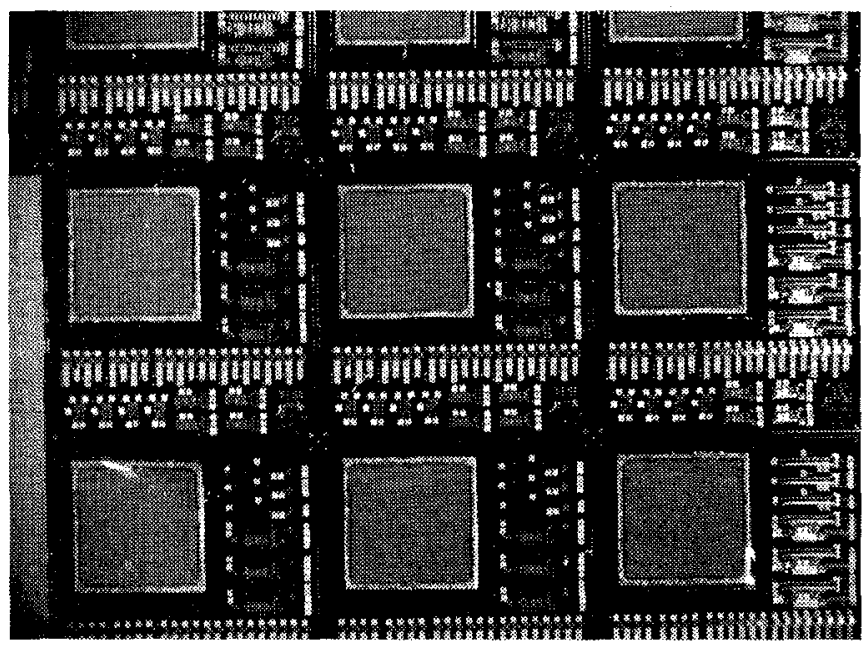

Figure 3: Photograph of nine polyimide microphones with integrated CMOS preamplifiers. The size of each chip is $5 \times 5$ $m m$
The $3-\mathrm{dB}$ cut-off frequency of the microphone is $2.3 \mathrm{kHz}$, and the collapse voltage was measured to be $6 \mathrm{~V}$. The bandwidth and the sensitivity of the microphone can be increased by increasing the air gap and/or the diaphragm thickness. To increase any of these parameters, however, a higher DC bias voltage is required. This can be achieved with a DC-DC voltage converter circuit, which has already been realised on the CMOS chip, and which can provide voltages of up to $18 \mathrm{~V}$ from a 2.4 $\mathrm{V}$ power supply.

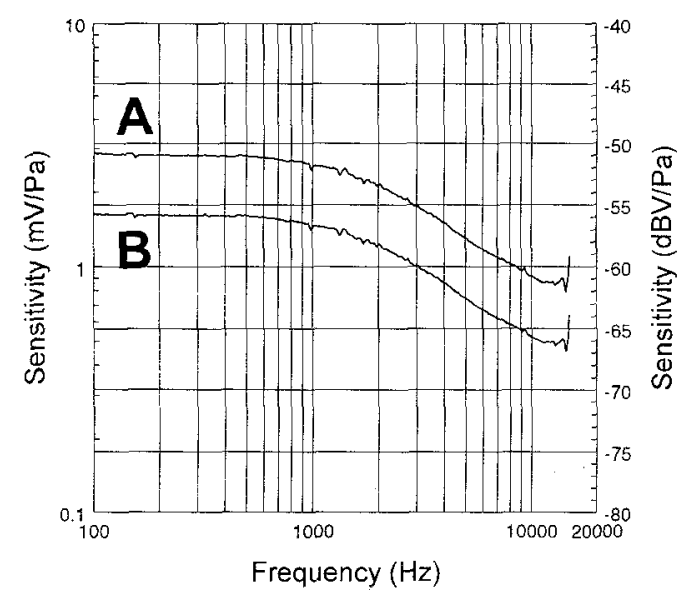

Figure 4: Measured frequency response of the integrated microphone at a DC bias voltage of $4 V$. (A) Integrated preamp. (B) External pre-amp.

\section{CONCLUSIONS}

A high performance integrated condenser microphone has been realised by performing a micromachining process on a completed CMOS substrate. The polyimide structure can be produced without harming the electronic circuits, and has proven to provide microphones with excellent properties, regarding noise level and sensitivity.

Currently, integrated microphones with increased air gap and diaphragm thickness are being realised, providing a wider bandwidth and higher sensitivity. The performance of these devices are expected to be sufficient for hearing aid applications (ENL $<24 \mathrm{~dB}(\mathrm{~A})$ SPL).

\section{ACKNOWLEDGEMENTS}

Research sponsored by the Dutch Technical Foundation STW.

\section{REFERENCES}

[1] M. Pedersen, W. Olthuis \& P. Bergveld, "A silicon condenser microphone with polyimide diaphragm and backplate," Accepted for publication in Sensors and Actuators $A$.

[2] M. Pedersen, R. Schellin, W. Olthuis \& P. Bergveld, "Electroacoustical measurements of silicon microphones on wafer scale," To appear in Journal of the Acoustical Society of America, 101 April (1997). 